\title{
Independently Owned Blood Products Testing Facility
}

National Cancer Institute

\section{Source}

National Cancer Institute. Independently Owned Blood Products Testing Facility. NCI

Thesaurus. Code C133331.

A separately operated and owned facility that performs routine blood and plasma donor testing. 